ISSN: 2600-5859

\title{
Educación y organización como impulsores de la creatividad y la innovación en el turismo
}

\begin{abstract}
(c) (i) () (2)
\end{abstract}
Education and organisation as drivers of creativity and innovation in tourism

Dr.C. Roberto M. Llanes Pérez. ${ }^{1}$ \& MSc. Yureidys García Leonard. ${ }^{2}$

Recibido: 07-12-2019 / Revisado: 08-12-2019 / Aceptado: 11-12-2019 / Publicado: 03-01-2020

Resumen. $\quad$ DOI: $\underline{\text { https://doi.org/10.33262/concienciadigital.v3i1.996 }}$

La educación, por una parte, como formadora de los técnicos, especialistas y profesionales, agentes principales de la sociedad y de su desarrollo y por otra parte, la organización de las asociaciones en empresas, instituciones y entidades de todo tipo que dichos agentes conforman para su convivencia y desarrollo colectivo en la sociedad, constituyen elementos impulsores de la creatividad y la innovación. En la ponencia se profundiza en estos elementos que unidos a la realidad cubana exponen estrategias importantes para que dicha realidad, en la actividad turística, mejore sustancialmente, dando una mayor respuesta a los cambios que se producen en las tecnologías de la información y en la comunicación.

Palabras claves: educación, organización, creatividad, innovación.

\section{Abstract.}

Education, on the one hand, as a trainer of technicians, specialists and professionals, the main agents of society and its development and, on the other hand, the organisation of associations in companies, institutions and entities of all kinds that these agents make up for their coexistence and collective development in society, are driving forces for creativity and innovation. The paper delves into these elements that together with the Cuban reality expose important strategies so that this reality, in the tourist activity,

\footnotetext{
${ }^{1}$ Universidad de la Habana. La Habana, Cuba. roberto.llanes@ftur.uh.cu

${ }^{2}$ Universidad de la Habana. La Habana, Cuba. ygarcia@ftur.uh.cu
} 
improves substantially, giving a greater response to the changes that take place in the information technologies and in the communication.

Keywords: education, organization, creativity, innovation.

\section{Introducción.}

Es importante en los momentos actuales, la estrecha colaboración entre las universidades y las empresas, entidades e instituciones gubernamentales, la creación de alianzas estratégicas, el perfeccionamiento continuo del proceso educativo y de formación y fortalecimiento del conocimiento, así como la unión de las fuerzas académicas y las entidades en general en el perfeccionamiento de los procesos,dados los cambios en las tecnologías de la información y las comunicaciones, principalmente en el sector turístico. Se expone un resumen de la situación actual y las acciones estratégicas que deben seguirse para dar respuesta a estos cambios.

\section{Desarrollo.}

La formación de profesionales

La universidad es una institución que en el devenir histórico del desarrollo de las sociedades juega un rol esencial, el resurgir de los procesos de innovación, el conocimiento como un factor productivo más, impacta directamente en la universidad moderna, que en respuesta a las demandas de un mercado laboral cambiante y en constante evolución replantea su misión, a lo que varios autores del tema la asocia a la responsabilidad ante la sociedad de formar y convertirse así misma, en un actor activo, encargada de generar, transferir conocimiento y crear profesionales.

Esto requiere que sus procesos sustantivos se interrelacionen tomando a la investigación como el proceso que articule a la docencia y a la extensión de manera que converja en lo que Rojas y Aguirre (2015) denominan "universidades de investigación", fomentar en los profesionales una cultura investigativa que a juicio de Maldonado (2007) tiene que ser ideológicamente critica, autónoma y capaz de adherirse a los adelantos del conocimiento.

Se destaca que la universidad tiene la oportunidad de realizar reformas, donde el estudiante no solo aprenda en el espacio universitario, sino que convive en su autoaprendizaje múltiples experiencias educativas, donde el componente práctico es importante. Se plantea que dentro de este marco se presentan retos de los que cabe destacar: un cambio de mentalidad en lo metodológico y la docencia basada en un sistema de aprendizaje, características beneficiosas así como la dimensión social que se le otorga a la universidad. Se reconoce que la 
contribución de la universidad debe estar guiada por la relación entre la formación y las demandas del entorno y las necesidades sociales.

La educación desde sus inicios debe poseer las herramientas pedagógicas para desarrollar un proceso de enseñanza-aprendizaje en el cual los estudiantes participan en su formación, realizan trabajos independientes y colectivos sobre contenidos temáticos y esencialmente deben centrarse en el proceso de razonamiento en la comprensión de dichos contenidos y en la formación del conocimiento. Se debe además incitar, a la aplicación de formas elementales de la creatividad y la investigación por parte de los estudiantes.

En los estudios superiores, esta participación de los estudiantes se amplía y profundiza en las temáticas relacionadas con su formación profesional, realizando prácticas en empresas e industrias o establecimientos en general, ya sean de producción o de servicios, y participando en investigaciones bajo la tutoría de profesores.

Estos estudiantes se transforman en profesionales, incorporados a empresas, entidades e instituciones gubernamentales y asociaciones, que conjuntamente con técnicos y especialistas, conforman la fuerza de trabajo de la sociedad. Pero, las sociedades, en su carácter dinámico y en su desarrollo, van introduciendo avances tecnológicos necesarios, producto de los estudios e investigaciones, en los cuales participa el espíritu creativo e innovador.

Estos esfuerzos y resultados en investigación, conocimiento y tecnología, también conllevan a un cambio simultáneo en los procesos y políticas de organización del trabajo, en las estructuras y funcionalidades de las instituciones y también en la formación de los recursos humanos.Río Cobián, (2003)

Estos cambios simultáneos en los procesos y políticas de organización del trabajo, en muchas ocasiones no se producen, existiendo desajustes organizacionales, lo cual impide el máximo aprovechamiento de estos cambios tecnológicos.

Las tecnologías de la información y la comunicación y la cultura e innovaciones de organización

La cultura organizacional es un sistema de normas, hábitos, creencias, valores y experiencias, que de una forma u otra son compartidos por las personas y/o grupos que conforman una institución, negocio, empresa o corporación y que se reflejan en la imagen de la misma, caracterizando un comportamiento de su gestión y de sus relaciones interpersonales.

Las normas son declaraciones de conductas y procedimientos en situaciones determinadas que deben cumplirse obligatoriamente por todos los integrantes de la organización, dentro de 
las cuales también se incluyen las referidas a los clientes, proveedores y toda persona no perteneciente a la organización pero que se relaciona con ella. Generalmente, las normas se agrupan en manuales de gestión y organización, siendo su cumplimiento y respeto obligatorio para todos.

Los hábitos son comportamientos estables en las actividades que se realizan individualmente o en grupos, derivados del cumplimiento de las normas o de experiencias individuales o grupales en la vida laboral y social de los integrantes de la organización.

Los valores son cualidades personales o grupales, tales como simpatía, honradez, cooperación, solidaridad, moral, etc.

La cultura organizacional es importantísima para una empresa o cualquier tipo de organización ya que refleja cualitativamente resultados de su gestión y comportamiento mostrando interna y externamente su comportamiento en relación a la gestión y las relaciones interpersonales.

La introducción de las tecnologías, principalmente las tecnologías de la información y la comunicación y el desarrollo gradual, social y estructural, de las sociedades que se encuentran en transformación a sociedades basadas en el conocimiento, crea nuevos sectores y transforma otros, eleva la utilización de altas tecnologías y del equipamiento correspondiente, se crean nuevas actividades de servicio y se fortalecen otras ya existentes, se amplía el comercio, la inversión y la colaboración internacional. Pero estas transformaciones requieren que los trabajadores y la población en general, eleven continuamente sus conocimientos y habilidades en estas tecnologías. La cultura organizacional se enriquece con estos nuevos conocimientos y habilidades en estas tecnologías.

Estos cambios elevan el nivel de competitividad de las empresas y fortalecen las instituciones ajenas almundo empresarial: la administración pública, la protección social, la cultura, los servicios a la población, el turismo y las académicas y de investigación, siendo estas últimas las de mayor importancia al ser las responsables de la formación y capacitación de profesionales, por lo que deben estar preparadas en instalaciones y en el equipamiento de dichas tecnologías de punta. Jacob, Marta y otros (2008).

Las innovaciones de organización mejoran la productividad y la calidad de la producción y los servicios y pueden estimular innovaciones en las nuevas situaciones futuras.

Estas innovaciones organizativas, en los casos que se produzcan internamente, se deben a las mejoras o novedades en la estructura y funcionalidades de los procesos y actividades propias de la empresa y en los casos que se produzcan externamente se deben a las nuevas relaciones 
con otros agentes, como la población y territorio anexo a la instalación, alianzas estratégicas, etc.

En base a esta clasificación, la innovación en servicios se puede definir como la conversión de ideas en productos, procesos o servicios que el mercado valora. Estas ideas pueden ser tecnológicas, comerciales y organizacionales. Esta definición debe ser entendida ensu sentido amplio, porque cubre todas las actividades que conllevan un cambio sustancialen la forma de hacer las cosas, incluyendo los servicios y productos que la empresa ofrece,así como la forma de producirlos, comercializarlos, distribuirlos y organizarlos.

Por tanto, las innovaciones tecnológicas son servicios nuevos o mejorados debido a la tecnología mientras que las innovaciones no tecnológicas consisten en nuevas formas de organización y gestión de las empresas o nuevos comportamientos en el mercado.

Para las universidades, estos cambios en las tecnologías de la información y la comunicación y su repercusión en las organizaciones, estructuras y funciones de las entidades también precisan que el personal docente e investigativo debe estar altamente preparado en el manejo de estas tecnologías ya que estos conocimientos y habilidades debe transmitirlos a los estudiantes. Y surge la pregunta si la academia posee el equipamiento necesario y la preparación requerida para ello. ¿Cómo pueden las universidades transmitir estos conocimientos y habilidades si el equipamiento es insuficiente? Si los conocimientos correspondientes a estas tecnologías no lo poseen totalmente el claustro y, sin embargo las entidades poseen los equipamientos y manejan los programas de los sistemas de gestión adquiridos, ¿no existe una brecha tecnológica entre empresa y academia?

Situación actual de las empresas respecto a la innovación

Por otra parte (Blanco (2012-3), García y Hernández, 2010), Gutiérrez (2012) y Gutiérrez (2013), (Díaz, 2013; Cazull, Hernández y Sánchez, 2012) en investigaciones realizadas por estos autores, resultaron las conclusiones siguientes:

- Para algunas empresas y sectores, la innovación ha tomado el papel principal, sin embargo, otras empresas y sectores perciben la innovación como algo complejo y desconocido.

- Existencia de tecnologías obsoletas, problemas de gestión en la cadena de suministros, poca interacción con proveedores y clientes, la casi escasa vigilancia tecnológica, inexistencia de una cultura innovadora, políticas deficientes de motivación e incentivos, y preparación organizacional poco apta para la innovación.

- Marco regulatorio limitante e insuficiente impacto de la innovación. 
- Inexistencia de ambientes creativos para la incorporación de soluciones a las barreras existentes.

- Insuficiente avance del papel de la ciencia, la tecnología y la innovación.

- Carencia de estrategias de innovación articuladas con los objetivos y estrategias empresariales.

- Poco acceso a la información científica tecnológica actualizada.

- Débiles vínculos con universidades y centros de investigación.

- Dificultades para el financiamiento de la innovación, resistencia al cambio y exceso de centralización.

- Existe por las empresas una prioridad a los problemas urgentes y a las tareas de carácter operativo.

- Las bases normativas de los órganos reguladores que faciliten un conocimiento y competencias presentan espacios vacíos y en ocasiones promueven y premian de forma indefinida con distinciones a empresas como innovadoras o aspirantes a innovadoras.

- Insuficiente documentación de conocimientos y capacidades empresariales.

- Ausencia generalizada de sistemas de monitoreo de competidores y clientes

- No realización de estudios de mercado, lo que impide el conocimiento pleno de las necesidades y expectativas de los clientes actuales y potenciales como fuente de procesos de innovación.

- Se desconocen los impactos de los procesos de innovación y la evaluación de sus resultados.

- No hay integración respecto a los procesos de la empresa y al de innovación.

- No existen encadenamientos con otros actores: clientes, universidades, centros de investigación, etc.

¿Es la innovación imprescindible para mantener la competitividad? No, se puede ser competitivo durante un tiempo sin ser innovador, con sólo mantener sistemas de mejora continua. No obstante, el mantener mejoras continuas ante barreras u obstáculos a la innovación por problemas financieros o de otra índole, es también innovación, pues solo el transformar un proceso para obtener mejores resultados es una innovación organizacional o de proceso.

Para impulsar la innovación y creatividad en las entidades del turismo, la Plataforma Estratégica, en el marco de la cultura organizacional, debe estar dirigida al desarrollo de competencias organizativas y directivas. Una empresa es innovadora en la medida que aporta novedades a su mercado de referencia. 
La innovación se fundamenta en la creatividad, el conocimiento y la organización, en su funcionamiento para el logro de objetivos. La plataforma estratégica se concentra en los elementos siguientes:

Liderazgo: el líder tiene un papel clave como promotor de una empresa con una organización innovadora, donde el personal se encuentra preparado con los conocimientos requeridos para asumir actitudes creativas e innovadoras. La base de las ideas creativas nace del conocimiento. Es fundamental que los directivos y colaboradores tengan la capacidad de adaptarse a los cambios. El directivo debe generar, valorar, nutrir y apoyar la aplicación de ideas creativas y debe actuar como creadores de equipos o redes de apoyo al servicio de la innovación, dado que la innovación requiere competencias de grupo además de individuales, logrando consensuar las experiencias y opiniones de los mismos.

Estrategia líder de diferenciación: La creación de valor se enmarca en la excelencia operativa, liderazgo en producto y relaciones íntimas con los clientes. Cada estrategia aporta una propuesta de valor concreta para los clientes del mercado referencial y las empresas deben ser capaces de seleccionar cuál de ellas aporta la diferenciación más importante, la que se considere líder para los cambios y sobre la cual se generan o descansan el resto de las estrategias. Una estrategia es más innovadora en la medida que aporta mayor diferenciación a la empresa.

Dirección: Una cultura innovadora tiene sus rasgos propios. El diseño del sistema de dirección de la organización y del personal, la definición de puestos de trabajo y el estilo de dirección se orientan al desarrollo profesional y humano del personal, lo que incide en las habilidades creativas, los conocimientos y la motivación y el compromiso. En el desarrollo de competencias directivas es fundamental un sistema de evaluación que incluya competencias relativas a la creatividad que fomente habilidades tales como: la imaginación, la intuición y el pensamiento enriquecido (no convencional). También la resolución de problemas, la asignación de responsabilidades o el sistema de información y sus redes, son áreas importantes que inciden en el desarrollo de competencias para la innovación.

Gestión de los activos clave en la cadena de valor: Los activos clave en la cadena de valor son la tecnología, el conocimiento y las relaciones en toda la cadena de valor:

- En la gestión de la tecnología, se trata de los conocimientos aplicados en productos, procesos y tecnologías. La investigación básica debe realizarse por los centros de investigación y universidades, comités de expertos, colaboradores en los consejos científicos y en las áreas o direcciones organizacionales, etc. Las transferencias de tecnologías se realizan mediante acuerdos de colaboración, joinventures, inversiones, licencias, etc.

- Gestión del conocimiento: Los tipos relevantes de conocimientos para la innovación cubren aspectos que abarcan el mercado, los procesos de creación de nuevos productos, de producción 
y comercialización y los tecnológicos. Toda empresa puede acceder a conocimientos externos para mejorar la calidad de las ideas que genera. La generación, organización, desarrollo y distribución de los conocimientos, como procesos de gestión del conocimiento, deben estar en correspondencia con el tipo de innovación que se haya definido y también con los demás elementos que condicionan dicha innovación. El aprendizaje organizacional es factor clave en la gestión del conocimiento, jugando un papel importante los centros de investigación y las universidades y otros medios de adquisición de conocimientos.

- Gestión de las relaciones en la cadena de valor: Es importante integrar los proveedores clave en el diseño de los productos y servicios de la empresa, dado el impacto del mismo en el proceso de desarrollo de productos y servicios y su calidad, ya sea en el costo o en el tiempo de lanzamiento al mercado. Esta relación con los proveedores facilita el acceso a la información y conocimientos de ambas partes, no obstante esta relación debe basarse en alianzas estratégicas ya que esta dependencia disminuye la flexibilidad de aprovisionamiento.

- Proceso de innovación de nuevos productos y/o servicios: El proceso se inicia con la generación del producto y/o servicio, la cual está condicionada a criterios como el ciclo de vida, la rentabilidad actual y esperada, viabilidad técnica, etc., y finaliza con el producto y/o servicio puesto en el mercado.

- Desarrollo de nuevos productos: Es la secuencia de actividades que permite pasar del diseño del producto y/o servicio a su producción y comercialización de acuerdo a sus especificaciones mercadológicas. Esta secuencia define alrededor del $80 \%$ de los costos, aprovisionamiento de componentes, formas de distribución del producto, proyectos de investigación y desarrollo a elaborar, etc. El briefing, en la tarea de desarrollo, define resumidamente las condiciones que debe cumplir el nuevo producto y/o servicio (calidad, precio, recursos, tiempo de lanzamiento, objetivos comerciales y financieros (facturación, cuota de mercado, rentabilidad).

- Innovación en el proceso de producción: En este proceso se designa el responsable del proyecto, el presupuesto y los colaboradores internos o externos con la meta de reducción de costos, mejora de calidad, mayor flexibilidad, etc, participando los departamentos de márketing, calidad e ingeniería de productos, definiendo o redefiniendo los procesos.

- Innovación en el proceso de comercialización: Nuevas formas de comercialización aportan ventajas competitivas. Algunas empresas han conseguido diferenciar su oferta a través de la aplicación de las TIC"s en sus procesos de comercialización, mediante la redefinición de los procesos de pedidos y facturación, mejoramiento del sistema de gestión de quejas y de retención de clientes, el sistema de atención técnica y el sistema de posventa. El proceso de desarrollo de un nuevo producto va acompañado de una revisión del canal de distribución, la modalidad de venta y el servicio posventa.

- Definición de los resultados y el aprendizaje: Las áreas de resultado que se persiguen en innovación, se plasman en indicadores, facilitando el seguimiento de la buena marcha del proceso y el desarrollo de nuevas acciones correctivas cuando sea necesario. Las acciones 
resultantes de la investigación implicarán un nuevo orden organizacional y de participación que mediante el aprendizaje y el compromiso, la caracterizarán como empresa innovadora.

Principales acciones estratégicas

Hay muchas otras situaciones que se presentan en las empresas y que impiden de una u otra forma saltos cualitativos en los procesos de innovación: pocos intercambios entre especialistas nacionales y extranjeros, ineficiencias en los sistemas de comunicación interna y externa de las empresas, carencia de personal preparado en las nuevas tecnologías, envejecimiento del equipamiento, falta de proveedores, etc.

Los estudios realizados por estos autores, de amplio contenido, destacan:

1. Debe estabilizarse la permanencia de los directivos y personal responsabilizados con los procesos de innovación y conformarse una carpeta única de documentos relacionados con las normas, resoluciones y referencias, así como las herramientas y análisis de resultados e impactos de la gestión.

2. Debe desarrollarse, preferiblemente con las universidades, un sistema de preparación del personal, talleres, cursos y otros espacios de intercambio y superación.

3. Diseñar una plataforma uniforme y una base normativa y metodológica para la gestión de la innovación.

4. La gestión de la innovación debe estar articulada con los demás sistemas de gestión en un sistema integral de gestión.

5. Considerar la gestión de la innovación como factor de competitividad y elemento estratégico de la empresa.

6. Diseñar y aplicar un sistema de indicadores para la evaluación de la gestión de la innovación.

7. Entre los procesos de agregación de valor y la generación de conocimiento especializado debe asegurarse una estrecha relación. La articulación de un sistema que asegure la generación de valor en la empresa mediante procesos de generación de conocimiento especializado hacia lo interno de la empresa, lo que implica desplegar procesos de aprendizaje, adquisición y transferencia de conocimientos y hacia lo externo mediante alianzas estratégicas y nexos con instituciones internacionales.

8. Los sistemas de vigilancia e inteligencia empresarial son fundamentales para identificar oportunidades de innovación, promoviendo la articulación efectiva entre los procesos $\mathrm{I}+\mathrm{D}+\mathrm{i}$ y las estrategias empresariales.

9. La organización debe desarrollarse y ser consistente con los procesos de innovación, renovando las estructuras y los estilos de dirección, flexibilizando el sistema de gestión para accionar rápidamente ante cambios de todo tipo que puedan afectar la competitividad $\mathrm{u}$ otros aspectos en la gestión empresarial. 
10. Avanzar en el perfeccionamiento del marco regulatorio. Es necesario aplicar un marco regulatorio menos restrictivo para el funcionamiento de las empresas, que conceda mayor autonomía y capacidad de decisión acerca del manejo de sus recursos para desarrollar la gestión de la innovación y mejorar el sistema de incentivos a colectivos e individuos que innovan o contribuyen desde sus responsabilidades a la innovación.

A criterio de los autores, se perfilan avances en el marco regulatorio empresarial que propenden al fortalecimiento de la gestión de la innovación en las empresas. Entre ellos, la flexibilización de los objetos sociales de las empresas promueve el aprovechamiento de sus potencialidades en función de oportunidades del entorno mediante procesos de innovación. Asimismo, la posibilidad de emplear parte de las utilidades para el desarrollo de procesos de I+D y el incremento de salarios e incentivos, entre otros destinos, puede convertirse en un acicate a las empresas para lograr mejoras importantes en la gestión y resultados de la innovación.

\section{Tendencias.}

¿Qué se espera en lo adelante respecto a la gestión de la innovación en la gestión hotelera en Cuba? Lo primero es lograr los avances mínimos alcanzados internacionalmente teniendo en cuenta las limitaciones financieras y el bloqueo económico, financiero y operativo impuesto por los EE.UU. Lo segundo es perfeccionar lo que se tiene, innovar en la organización de los procesos, en los productos, mejorar la calidad, la atención al cliente y sus niveles de satisfacción, y tener en cuenta que también hay clientes que prefieren los servicios personalizados y no dependen tanto de la digitalización.

- Los programas para gestionar la actividad hotelera, los cuales deben ir perfeccionándose y ampliándose para lograr un mayor nivel de integración.

- La utilización de todos los medios digitales para el comercio electrónico orientado a nuevas tecnologías (marketing digital) que optimizan y hacen más fácil el tratamiento de la información y la relación con los potenciales clientes, para convertirlos en clientes y fidelizarlos.

- Tener diferentes canales de distribución, aprovechando la tecnología para combinarlos con el de venta directa. Ejemplo: GDS, portales en Internet, Redes Sociales, etc.

- Utilización de herramientas y programas de predicción que permitan pronosticar y diseñar escenarios respecto a las demandas del mercado, la estacionalidad y evolución de las variables más importantes que faciliten el cálculo de precios, ofertas y disponibilidad al futuro.

- Personalizar ofertas, adaptadas a las necesidades y preferencias de cada tipo de cliente, teniendo en cuenta la diversificación de la demanda.

- Utilizar las redes sociales en la promoción y en el conocimiento de las satisfacciones de los clientes, quienes lo comentan en las mismas. 
- La utilización de los sitios WEB debe ampliarse brindando una mayor interacción con los clientes y potenciales clientes. Un sitio WEB, moderno, intuitivo y bien diseñado atraerá más clientes y facilitará el diálogo directo con los clientes.

- Las aplicaciones para móviles aportarán posibilidades para la fidelización de los clientes, facilitarán las reservas y el conocimiento y disfrute de actividades culturales y gastronómicas y otras informaciones como localización, transporte, etc.

- Cada vez hay más cantidad de personas que utilizan sus móviles para planificar sus viajes, y que prefieren realizar las reservas de vuelos y hoteles desde el mismo móvil. Ofrecer este servicio al cliente por medio de una aplicación que permita conocer la disponibilidad del hotel, las tarifas, y realizar reservas en el momento ha empezado a ser de vital importancia.

- Prestar gratuitamente el servicio de Wi-Fi en el hotel facilitándole al cliente su conexión con los servicios del hotel y con el exterior. La gran popularidad de la conexión Wifi hará que los hoteles que ya ofrezcan este servicio de manera gratuita en todo el establecimiento tengan mayores posibilidades de conquistar nuevos clientes.

- Tener el personal capacitado en las novedades de las tecnologías de la información y comunicación y en su preparación permanente ante los cambios que se producirán en la demanda y en las tecnologías.

- Estas son algunas de las tendencias actuales en el sector hotelero, que seguramente seguirán evolucionando ya que el desarrollo no se detiene.

\section{Referencias bibliográficas.}

Blanco, H. (2013). Gestión de la Innovación en la empresa: Estudios de casos y propuestas de mejoramiento. En: Seminario Anual del Centro de Estudios de la Economía Cubana, (CEEC). Universidad de La Habana. ISBN: 978-959234-093-0

Blanco, H. (2012). Dirección Integrada de la Gestión Empresarial. Seminario Anual del CEEC. Universidad de La Habana. ISBN 978-959-234-085-5.

Cazull, M; G. Hernández, y N. Sánchez (2012). Sistema de Innovación Tecnológica: Estudio de Casos. En:http: //www.gestec.disaic.cu/ponencias_Cuba_2010.htm. (consulta: 10 de diciembre de 2012).

Díaz, I (2013): Desafíos de la Innovación Empresarial en Cuba. En: Seminario Anual del CEEC, Universidad de La Habana. ISBN: 978-959-234-093-0

García, Y. y G. Hernández (2010).Caracterización del proceso de innovación en la industria sidero-mecánica de Villa Clara. En: 
ISSN: 2600-5859

http://www.gestec.disaic.cu/ponencias-Cuba_2010.htm. (consultado: $10 \mathrm{de}$ diciembre de 2012).

Gutiérrez, O. (2013). "Reflexiones sobre los ambientes de innovación en las empresas cubanas". En: Seminario Anual del CEEC. Universidad de La Habana. ISBN: 978-959-234-093-0

Gutiérrez, O. (2012). “La contextualización de los modelos gerenciales y la formación de directivos empresariales en Cuba". En: Seminario Anual del CEEC. Universidad de La Habana. ISBN 978-959-234-085-5.

Jacob, Marta, Aguiló, Eugeni (2008), La innovación en el sector turístico. El caso de Baleares, Rotur, Revista de Ocio y Turismo, No 1 - pp. 51 - 64, ISSN: $1888-$ 6884, Coruña, Universitat de les Illes Balears

Maldonado, L. et al. (2007). Visibilidad y formación en investigación. Estrategias para el desarrollo de competencias investigativas. Studiositas, 2(2), 43-56.

Río Cobián, Eduardo del (2003) La importancia de la innovación organizativa para la obtención de los beneficios derivados de la introducción de las tecnologías de la información. [Tesis]. Facultad de Ciencias Económicas y empresariale, Universidad Complutense de Madrid.

Rojas, C. y Aguirre, S. (2015). La formación investigativa en la educación superior en América Latina y el Caribe: una aproximación a su estado del arte.

Revista Eleuthera, 12, 197-222. 10.17151/eleu.2015.12.11.

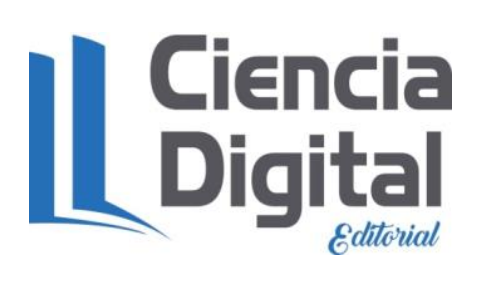


ISSN: 2600-5859

\section{Para citar el artículo indexado.}

Llanes Pérez, R., \& García Leonard, Y. (2020). Educación y organización como impulsores de la creatividad y la innovación en el turismo. ConcienciaDigital, 3(1), 23-35. https://doi.org/10.33262/concienciadigital.v3i1.996

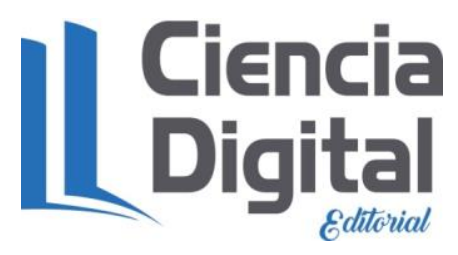

El artículo que se publica es de exclusiva responsabilidad de los autores y no necesariamente reflejan el pensamiento de la Revista Conciencia Digital.

El articulo queda en propiedad de la revista y, por tanto, su publicación parcial y/o total en otro medio tiene que ser autorizado por el director de la Revista Conciencia Digital.

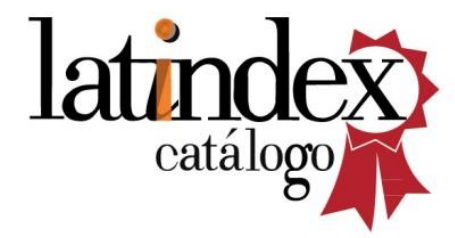

Conciencia

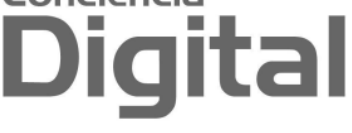

